\title{
Invariant Observer Applied to Anaerobic Digestion Model
}

\author{
Khadidja Chaib Draa, Holger Voos \\ Interdisciplinary Centre for Security, Reliability \\ and Trust (SnT) - Université du Luxembourg \\ L-1359, Luxembourg-Kirchberg, Luxembourg \\ khadidja.chaibdraa/holger.voos@uni.lu
}

\author{
Marouane Alma, Mohamed Darouach, \\ CRAN-CNRS UMR 7039 \\ Université de Lorraine, IUT de Longwy \\ 54400, Cosnes et Romain, France \\ marouane.alma/mohamed.darouach@univ-lorraine.fr
}

\begin{abstract}
In this note, we design an invariant observer for a two step (acidogenesis-methanogenesis) mass balance non linear model, in order to estimate simultaneously all bacteria and substrate concentrations found in the anaerobic digestion process. The particularity of the designed observer is the use of only the methane flow rate which is cheap to measure and commonly measured online even at industrial scale.
\end{abstract}

Index Terms-Non-Linear Models; Invariant Observer; Anaerobic Digestion.

\section{INTRODUCTION}

The Anaerobic Digestion (AD) is a promising process for waste valorisation and energy production. It consists on the transformation of organic matter into biogas, which is a mixture of gaseous, through several biologic reactions including different species of microorganisms. The produced biogas is then converted to electrical energy and injected to the power grid. Despite the advantages that can present the AD process it has still not found its place in industry due to the the lack of process knowledge. In fact, this goes back to the lack of sensors and the expensive price of the existing ones. To overcome this issue, a couple of software sensors has been designed. Among them, we can cite the asymptotic observer reported in [2] which is quite simple and does not require the knowledge of some specific non linear functions. However, it has a drawback concerning the speed of convergence which is equal to the control input. An extension of the asymptotic observers has been proposed in [3] which has the advantage of using reliable measurement, which are non linear functions of the state vector, to improve its estimation. The same authors have proposed interval observers to estimate the interval where the state is lying when the system has large uncertainties. However, generally the rate of convergence is not tunable. A new kind of observers has been proposed in [1] and [8] and applied to a class on chemical reactors, they are called invariant observers and are based on Lie group symmetries. The advantage of such observers is their adjustable and robust convergence as it has been shown in [5]. In the present work, we apply an invariant observer to a non linear model for the $\mathrm{AD}$ process with the only use of methane flow rate measurement, which is cheap and easy to do online. The present note is organised as the following: In Sec. (II) we

978-1-5090-1314-2/16\$31.00 (C) 2016 IEEE present the studied model, then in Sec. (III) we give brief notions about the invariant observer and the needed ingredients to design it. In Sec. (IV) we detail the designed observer and give the prove of convergence. Finally, after showing the simulation results in Sec. (V) we conclude the paper in Sec. (VI).

\section{MOdel Description}

The nonlinear model for the AD process reported in [6] considers the following two reactions as the limiting steps:

1) Acidogenesis with reaction rate $r_{1}=\mu_{1} x_{1}$ :

$$
k_{1} s_{1} \stackrel{r_{1}}{\rightarrow} x_{1}+k_{2} s_{2}+k_{4} \mathrm{co}_{2}
$$

2) Methanogenesis with reaction rate $r_{2}=\mu_{2} x_{2}$ :

$$
k_{3} s_{2} \stackrel{r_{2}}{\rightarrow} x_{2}+k_{5} \mathrm{CO}_{2}+k_{6} \mathrm{ch}_{4}
$$

where, $s_{1}\left(\mathrm{Kg} / \mathrm{m}^{3}\right)$ is the organic substrate degraded by the acidogenic bacteria $x_{1}\left(\mathrm{Kg} / \mathrm{m}^{3}\right)$ to volatile fatty acids $s_{2}$ $\left(\mathrm{mol} / \mathrm{m}^{3}\right)$ which are supposed to behave like pure acetate, and $x_{2}\left(\mathrm{Kg} / \mathrm{m}^{3}\right)$ is the methanogenic bacteria which consume $s_{2}$ and produce $\mathrm{CO}_{2}$ and $\mathrm{Ch}_{4}$. The substrates and microorganisms concentrations are modelled by the following equations:

$$
\begin{aligned}
& \dot{s_{1}}=D(t)\left(s_{1 i n}-s_{1}\right)-k_{1} \mu_{1}\left(s_{1}\right) x_{1} \\
& \dot{x_{1}}=\left(\mu_{1}\left(s_{1}\right)-\alpha D(t)\right) x_{1} \\
& \dot{s_{2}}=D(t)\left(s_{2 i n}-s_{2}\right)+k_{2} \mu_{1}\left(s_{1}\right) x_{1}-k_{3} \mu_{2}\left(s_{2}\right) x_{2} \\
& \dot{x_{2}}=\left(\mu_{2}\left(s_{2}\right)-\alpha D(t)\right) x_{2}
\end{aligned}
$$

with:

$$
\left\{\begin{array}{llc}
\mu_{1}\left(s_{1}\right) & = & \mu_{1 \max } \frac{s_{1}}{s_{1}+k_{s_{1}}} \\
\mu_{2}\left(s_{2}\right) & = & \mu_{2 \max } \frac{s_{2}}{s_{2}+k_{s_{2}}+\left(\frac{s_{2}}{k_{i_{2}}}\right)^{2}}
\end{array}\right.
$$

where, $s_{1 i n}$ and $s_{2 i n}$ are the input concentrations of $s_{1}$ and $s_{2}$ respectively, $D\left(\frac{1}{d a y}\right)>0$ is the dilution rate and is the control variable, $k_{i}$ are the yield coefficients defined in Table (I) with the other parameters. In this work we take $\alpha=1$ which corresponds to an ideal Continuous Stirred Tank Reactor (CSTR) [6], and we choose the output to be the methane gas flow rate:

$$
y=k_{6} \mu_{2}\left(s_{2}\right) x_{2}
$$


Before giving the structure of the designed observer for the system (3), we repport in the next section a brief introduction to the invariant observers.

TABLE I: Model Parameters

\begin{tabular}{lll}
\hline \hline Acronyms & Definition & Units \\
\hline$k_{1}$ & Yield for $s_{1}$ degradation & $\mathrm{Kg} \mathrm{COD} / \mathrm{Kg} x_{1}$ \\
$k_{2}$ & Yield for $s_{2}$ production & $\mathrm{mol} \mathrm{VFA} / \mathrm{Kg} x_{1}$ \\
$k_{3}$ & Yield for $s_{2}$ consumption & $\mathrm{mol} \mathrm{VFA} / \mathrm{Kg} x_{2}$ \\
$k_{6}$ & Yield for $c h_{4}$ production & $\mathrm{mol} C H_{4} / \mathrm{Kg} x_{2}$ \\
$\mu_{1 \max }$ & Maximum $x_{1}$ growth rate & $1 /$ day \\
$\mu_{2 \max }$ & Maximum $x_{2}$ growth rate & $1 / d a y$ \\
$k_{s 1}$ & Half saturation constant associated to $s_{1}$ & $\mathrm{Kg} \mathrm{COD} / \mathrm{m}^{3}$ \\
$k_{s 2}$ & Half saturation constant associated to $s_{2}$ & $\mathrm{~mol} \mathrm{VFA} / \mathrm{m}^{3}$ \\
$k_{i 2}$ & Inhibition constant associated to $s_{2}$ & $\left(\mathrm{~mol} \mathrm{VFA} / \mathrm{m}^{3}\right)^{\frac{1}{2}}$ \\
\hline \hline
\end{tabular}

\section{INVARIANT SYSTEM AND OBSERVER [5]}

In the present work the invariance refers to the invariance under a group action. Hereafter, we give some notions about this concept but for more comprehension the reader is referred to [4], [8] and for more details to [7].

Let consider the system (9) and let $G$ be a Lie group of transformations which acts on $X$ by $\varphi_{g}: X \rightarrow X \quad \forall g \in G, \varphi_{g}$ is a diffeomorphism (at least $C^{1}$ ) on $X$ with $\left(\varphi_{g}\right)^{-1}=\varphi_{g^{-1}}$ and $\varphi_{g_{1}} \circ \varphi_{g_{2}}=\varphi_{g_{1} . g_{2}}$. Moreover, let's note the action of the group $G$ on $U$ by $\left(\psi_{g}\right)_{g \in G}$ and on $Y$ by $\left(\rho_{g}\right)_{g \in G}$.

Definition 1. $G$ is a symmetry group of (9) if for every solution $(x(t), u(t))$ of (9) and $\forall g \in G,\left(\varphi_{g}(x(t)), \psi_{g}(u(t))\right)$ is also a solution.

Therefore, the system (9) is said to be invariant under $G$ if and only if $\forall g, x$ and $u$ :

$$
f\left(\varphi_{g}(x), \psi_{g}(u)=D_{\varphi_{g}}(x) f(x, u)\right.
$$

where $D_{\varphi_{g}}$ is the Jacobian matrix of $\varphi_{g}(x)$.

After finding the group of transformation, one can write the following pre-observer for the system (9):

$$
\dot{\hat{x}}=F(\hat{x}, u, \hat{y})
$$

if and only if $\forall x$ and $u$ :

$$
F(x, u, h(x, u))=f(x, u)
$$

Moreover, the pre-observer (5) is said to be invariant if and only if $\forall g, \hat{x}$ and $\hat{y}$ :

$$
F\left(\varphi_{g}(\hat{x}), \psi_{g}(u), \rho_{g}(\hat{y})\right)=D_{\varphi_{g}}(\hat{x}(t) F(\hat{x}, u, \hat{y})
$$

To design an invariant observer, we need invariant functions and invariant vector fields:

- A function defined on $X \subset R^{n}$ is invariant if and only if: $J\left(\varphi_{g}(x)\right)=J(x), \forall g$ and $x$.

- A vector field $\omega$ is invariant with respect to the action of $\varphi_{g}$ on $X$ if and only if: $\omega\left(\varphi_{g}(x)\right)=D_{\varphi_{g}}(x) \omega(x), \forall g$ and $x$.
Finally, it has been proven in [8] that the general form of an invariant pre-observer for the system (9) is given by:

$$
\dot{\hat{x}}=f(\hat{x})+\sigma_{i} J_{i}(\hat{x}, y) \omega_{i}(\hat{x})
$$

with $J_{i}$ being an invariant function satisfying $J_{i}(\hat{x}, h(\hat{x}, u))=$ 0 and $\omega_{i}$ an invariant vector field. Moreover if (8) converges to (9) then it is called invariant observer.

\section{OBSERVER DESIGN}

Before designing any kind of observer, one has to check first the observability of the system. To do so, we have used the rank criterium. Unfortunately, for lack of space, we have not included calculations in this note. However, we recall here below the used criterium [4]:

Rank Criterium: The non linear system:

$$
\left\{\begin{array}{l}
\dot{x}=f(x, u) \\
y=h(x, u)
\end{array}\right.
$$

where $x \in X \subset R^{n}$ is the state vector, $u \in U \subset R^{m}$ the input and $y \in Y \subset R^{p}$ is the measured output is observable if:

$$
\operatorname{rank}\left(d h, d L_{f} h, \ldots, d L_{f}^{n-1} h\right)^{T}=n
$$

where $L_{f} h$ is the Lie derivative of $h$ along $f$.

$$
L_{f} h=\sum_{i=1}^{n} f_{i}(x) \frac{\partial h}{\partial x_{i}}
$$

and $d L_{f}^{k} h$ given by:

$$
d L_{f}^{k} h=\left[\begin{array}{llll}
\frac{\partial L_{f}^{k} h}{\partial x_{1}} & \frac{\partial L_{f}^{k} h}{\partial x_{2}} & \ldots & \frac{\partial L_{f}^{k} h}{\partial x_{n}}
\end{array}\right]
$$

For the system (3), rank $\left(d h, d L_{f} h, \ldots, d L_{f}^{n-1} h\right)^{T}=4$, and thus the system is observable.

Now, after the system observability has been checked, we use the invariant functions and vector fields given in [4], to design the following invariant observer for system (3):

$$
\begin{aligned}
\dot{\hat{s}}_{1}= & D(t)\left(s_{1 i n}-\hat{s}_{1}\right)-k_{1} \mu_{1}\left(\hat{s}_{1}\right) \hat{x}_{1} \\
& +a_{1}\left(\ln \left(\frac{y}{\hat{x_{2}}}\right)-\ln \left(\frac{\hat{y}}{\hat{x_{2}}}\right)\right) \\
\dot{\hat{x}}_{1}= & \left(\mu_{1}\left(\hat{s}_{1}\right)-D(t)\right) \hat{x}_{1}+a_{2}\left(\ln \left(\frac{y}{\hat{x_{2}}}\right)-\ln \left(\frac{\hat{y}}{\hat{x_{2}}}\right)\right) \\
\dot{\hat{s}}_{2}= & (t)\left(s_{2 i n}-\hat{s}_{2}\right)+k_{2} \mu_{1}\left(\hat{s}_{1}\right) \hat{x}_{1}-k_{3} \mu_{2}\left(\hat{s}_{2}\right) \hat{x}_{2} \\
& +a_{3}\left(\ln \left(\frac{y}{\hat{x_{2}}}\right)-\ln \left(\frac{\hat{y}}{\hat{x_{2}}}\right)\right) \\
\dot{\hat{x}}_{2}= & \left(\mu_{2}\left(\hat{s}_{2}\right)-D(t)\right) \hat{x}_{2}+a_{4}\left(\ln \left(\frac{y}{\hat{x_{2}}}\right)-\ln \left(\frac{\hat{y}}{\hat{x_{2}}}\right)\right)
\end{aligned}
$$

with:

$\left\{\begin{array}{llc}\mu_{1}\left(\hat{s}_{1}\right) & = & \mu_{1 \max } \frac{\hat{s}_{1}}{\hat{s}_{1}+k_{s_{1}}} \\ \mu_{2}\left(\hat{s}_{2}\right) & = & \mu_{2 \max } \frac{\hat{s}_{2}}{\hat{s}_{2}+k_{s_{2}}+\left(\frac{\hat{s}_{2}}{k_{i_{2}}}\right)^{2}}\end{array}\right.$

and:

$$
\hat{y}=k_{6} \mu_{2}\left(\hat{s}_{2}\right) \hat{x}_{2}
$$


Defining the errors: $e_{1}=\ln \left(s_{1}\right)-\ln \left(\hat{s}_{1}\right), e_{2}=\ln \left(x_{1}\right)-$ $\ln \left(\hat{x}_{1}\right), e_{3}=\ln \left(s_{2}\right)-\ln \left(\hat{s}_{2}\right), e_{4}=\ln \left(x_{2}\right)-\ln \left(\hat{x}_{2}\right)$ and using Eqs. $(3,4,10)$ and $(11)$ we obtain the following:

$$
\dot{e}=A(t) e+\Phi(t, e)
$$

with $e=\left[e_{1}, e_{2}, e_{3}, e_{4}\right]^{T}, A(t)$ the linear part of the error system:

$A=\left(\begin{array}{cccc}-v_{1}+v_{2} & -v_{3} & -a_{1} & -a_{1} \\ v_{4} & 0 & -a_{2} & -a_{2} \\ -v_{6}+v_{7} & v_{7} & A_{33} & -\left(a_{3}+v_{9}\right) \\ 0 & 0 & -a_{4}+v_{11}-v_{12} & -a_{4}\end{array}\right)$

where $A_{33}=-a_{3}-v_{5}-v_{7}+v_{8}+v_{10}$, and $\Phi(t, e)=\left[\Phi_{1}, \Phi_{2}, \Phi_{3}, \Phi_{4}\right]^{T}$ the non linear part:

$$
\begin{aligned}
\Phi_{1}= & -v_{1}\left(\mathrm{e}^{e_{1}}-e_{1}-1\right)-v_{2}\left[f_{1}\left(\mathrm{e}^{-e_{1}}+e_{1}-1\right)\right. \\
& \left.+f_{2}\left(\mathrm{e}^{-e_{1}}-1\right) e_{1}\right]+v_{3}\left[f_{1}\left(\mathrm{e}^{-e_{2}}+e_{2}-1\right)\right. \\
& \left.+f_{2}\left(\mathrm{e}^{-e_{1}}-1\right) e_{2}\right]-a_{1} \ln (g) \\
\Phi_{2}= & -v_{4}\left[f_{1}\left(\mathrm{e}^{-e_{1}}+e_{1}-1\right)+f_{2}\left(\mathrm{e}^{-e_{1}}-1\right) e_{1}\right] \\
& -a_{2} \ln (g) \\
\Phi_{3}= & -v_{5}\left(\mathrm{e}^{e_{3}}-e_{3}-1\right)+v_{6}\left[f_{1}\left(\mathrm{e}^{-e_{1}}+e_{1}-1\right)\right. \\
& \left.+f_{2}\left(\mathrm{e}^{-e_{1}}-1\right) e_{1}\right]-v_{7}\left[f _ { 1 } \left(\mathrm{e}^{-e_{1}-e_{2}+e_{3}}+\left(e_{1}\right.\right.\right. \\
& \left.\left.\left.+e_{2}-e_{3}\right)-1\right)+f_{2}\left(\mathrm{e}^{-e_{1}}-1\right)\left(e_{1}+e_{2}-e_{3}\right)\right] \\
& -v_{8}\left[f_{3}\left(\mathrm{e}^{-e_{3}}+e_{3}-1\right)+f_{4}\left(\mathrm{e}^{-e_{3}}-1\right) e_{3}\right. \\
& \left.+f_{5}\left(\mathrm{e}^{-2 e_{3}}-1\right) e_{3}\right]-v_{10}\left[f_{3}\left(\mathrm{e}^{-2 e_{3}}+2 e_{3}-1\right)\right. \\
& \left.+2 f_{4}\left(\mathrm{e}^{-e_{3}}-1\right) e_{3}+2 f_{5}\left(\mathrm{e}^{-2 e_{3}}-1\right) e_{3}\right] \\
& +v_{9}\left[f_{3}\left(\mathrm{e}^{-e_{4}}+e_{4}-1\right)+f_{4}\left(\mathrm{e}^{-e_{3}}-1\right) e_{4}\right. \\
& \left.+f_{5}\left(\mathrm{e}^{-2 e_{3}}-1\right) e_{4}\right]-a_{3} \ln (g) \\
\Phi_{4}(g) & -v_{11}\left[f_{3}\left(\mathrm{e}^{-e_{3}}+e_{3}-1\right)+f_{4}\left(\mathrm{e}^{-e_{3}}-1\right) e_{3}\right. \\
& \left.+f_{5}\left(\mathrm{e}^{-2 e_{3}}-1\right) e_{3}\right]+v_{12}\left[f_{3}\left(\mathrm{e}^{-2 e_{3}}+2 e_{3}-1\right)\right. \\
& \left.+f_{4}\left(\mathrm{e}^{-e_{3}}-1\right) 2 e_{3}+f_{5}\left(e^{-2 e_{3}}-1\right) 2 e_{3}\right] \\
& -a_{4} \ln (g)
\end{aligned}
$$

Since the error equations (12) are partitioned into a linear and a non linear part w.r.t the error, we prove the asymptotic stability of the origin using the following theorem:

Theorem 1. [4] Let's the linear system in $R^{n}$ :

$$
\dot{x}=A(t) x
$$

be uniformly asymptotically stable for $t_{0} \geq T$.

Lest's:

$$
\Phi: R \times R^{d} \rightarrow R^{d} \quad(t, x) \mapsto \Phi(t, x)
$$

be a continuous function and $\Phi(t, 0)=0$ such that:

$$
\forall \epsilon>0, \exists \delta_{\epsilon}>0:\|x\| \leq \delta_{\epsilon} \Rightarrow\|\Phi(t, x)\| \leq \epsilon\|x\|, \forall t \geq T
$$

Let's the following system hold:

$$
\dot{x}=A(t) x+\Phi(t, x)
$$

So the solution $x=0$ for the system (II) is uniformly asymptotically stable for $t_{0} \geq T$.

After the theorem has been announced, which is concerned with the asymptotic convergence of the error to the origin, we discuss in the following how it can be applied to the system
(12) in order to find the observer gains. Therefore, we start by proving the asymptotic stability of the linear part.

Let's take the classic Lyapunov equation:

$$
V=e^{T} P e
$$

where $P$ is a symmetric positive definite matrix. Before deriving the lyapunov equation, we rewrite the matrix $A$ in Eq. (12) as $A=\bar{A}-K C$, where:

$\bar{A}=\left(\begin{array}{cccc}-v_{1}+v_{2} & -v_{3} & 0 & 0 \\ v_{4} & 0 & 0 & 0 \\ -v_{6}+v_{7} & v_{7} & -v_{5}-v_{7}+v_{8}+v_{10} & -v_{9} \\ 0 & 0 & v_{11}-v_{12} & 0\end{array}\right)$,

$K=\left[a_{1}, a_{2}, a_{3}, a_{4}\right]^{T}$,

$C=[0,0,1,1]$

Thus the derivative of $V$ is:

$$
\dot{V}=e^{T} \underbrace{\left((\bar{A}-K C)^{T} P+P(\bar{A}-K C)\right)}_{-Q} e
$$

Therefore, the gain $K$ can be found by solving an $L M I$ problem $(Q>0)$ at the vertices of the matrix $\bar{A}$.

Concerning the non linear part of the system (12), it can be easily seen that $\Phi(t, 0)=0$. Moreover, the function $\Phi(t, e)$ is continuous and differentiable. Thus, using the differential mean value theorem we can write:

$$
\Phi(t, e)=\frac{\partial \Phi(t, z)}{\partial e} \quad e
$$

and moreover, we have:

$$
\|\Phi(t, e)\| \leq \underbrace{\left\|\frac{\partial \Phi(t, z)}{\partial e}\right\|}_{\epsilon}\|e\|
$$

Thus $\forall \epsilon>0, \exists \delta_{\epsilon}>0:\|e\| \leq \delta_{\epsilon} \Rightarrow\|\Phi(t, e)\| \leq \epsilon\|e\|, \forall t \geq$ $T$. Hence, the proof of the asymptotic stability of the origin for the system (12) has been completed.

\section{Simulation Results}

For simulation, we have taken $k_{1}=6.6, k_{2}=7.8$, $k_{3}=611.2, k_{6}=1139.2, k_{s 1}=4.95, k_{s 2}=9.28$, $k_{i 2}=20, \mu_{1 \max }=1.2, \mu_{2 \max }=0.69, s_{1 i n}=15$ and $s_{2 \text { in }}=80$. Furthermore, we have initialized the model at: $s_{1}(0)=4, x_{1}(0)=3.5, s_{2}(0)=15$ and $x_{2}(0)=0.3$, and the observer at: $\hat{s}_{1}(0)=5.5, \hat{x}_{1}(0)=3, \hat{s}_{2}(0)=13$ and $\hat{x}_{2}(0)=0.5$. Moreover, the simulation was carried out over 75 (days) with a varying input $D(t)$ as depicted in Fig (1). The obtained observer gain from the resolution of the $L M I$ problem $(Q>0)$ is $K=[-15,-50,120,500]^{T}$.

The simulation results are plotted in figures $(2,3,4)$ and (5). As it can be seen from the former figures, the designed observer is rapidly converging to the system state w.r.t the process dynamic. Moreover, we note that the addition of correction in the first two states $\left(s_{1}\right.$ and $\left.x_{1}\right)$ allow the observer to be faster than the one proposed in [5]. 


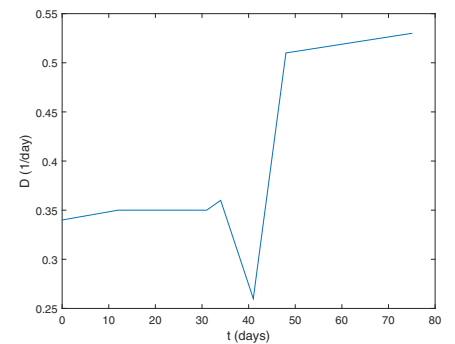

Fig. 1: Control Input $D(t)(1 /$ day $)$.

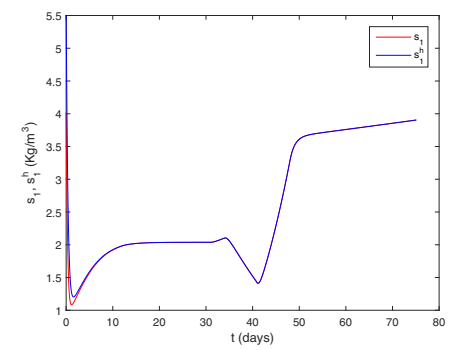

Fig. 2: Substrate $s_{1}$ and its estimation $\hat{s}_{1}\left(K g / m^{3}\right)$.

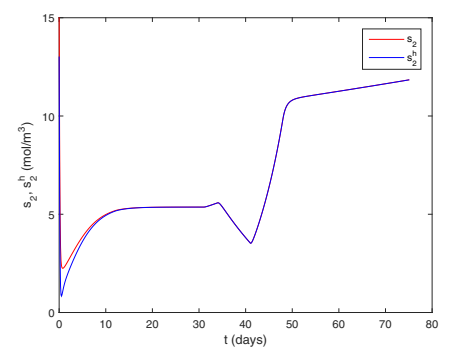

Fig. 3: Acids $s_{2}$ and its estimation $\hat{s}_{2}\left(\mathrm{~mol} / \mathrm{m}^{3}\right)$.

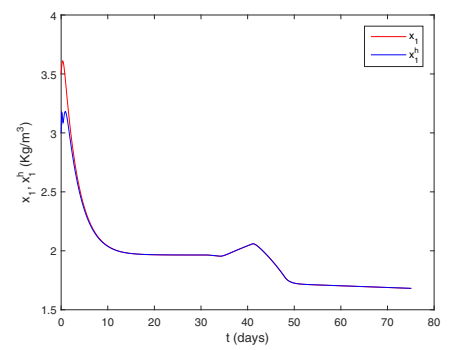

Fig. 4: Bacteria $x_{1}$ and its estimation $\hat{x}_{1}\left(\mathrm{Kg} / \mathrm{m}^{3}\right)$.

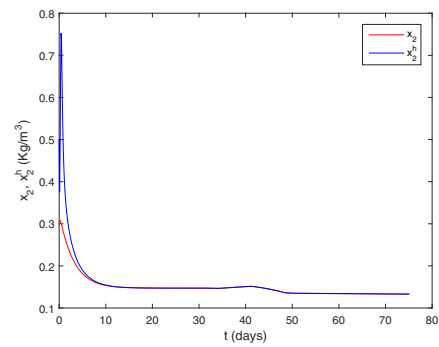

Fig. 5: Bacteria $x_{2}$ and its estimation $\hat{x}_{2}\left(K g / \mathrm{m}^{3}\right)$.

\section{CONCLUSION}

In this note, we have designed an invariant observer for the anaerobic digestion process with the use of an only one cheap and commonly done measurement at the industrial scale, which is the methane flow rate. The designed invariant observer has shown satisfactory behaviour and we target in the near future to evaluate its performance in the presence of noise in measurements, and finally use it for control in order to enhance the anaerobic digestion process.

$$
\begin{aligned}
& v_{1}=\frac{D(t) s_{1 i n}}{s_{1}} \quad v_{8}=\frac{k_{3} \mu_{2 \max } s_{2} x_{2}}{\left(s_{2}+k_{s_{2}}+\left(\frac{s_{2}}{k_{i_{2}}}\right)^{2}\right)^{2}} \\
& v_{2}=\frac{k_{1} \mu_{1 \max } s_{1} x_{1}}{\left(s_{1}+k_{s_{1}}\right)^{2}} \quad v_{9}=\frac{k_{3} \mu_{2 \max } x_{2}}{s_{2}+k_{s_{2}}+\left(\frac{s_{2}}{k_{i_{2}}}\right)^{2}} \\
& v_{3}=\frac{k_{1} \mu_{1 \max } x_{1}}{\left(s_{1}+k_{s_{1}}\right)} \quad v_{10}=\frac{2 k_{3} \mu_{2 \max } s_{2}^{2} x_{2}}{k_{i_{2}}^{2}\left(s_{2}+k_{s_{2}}+\left(\frac{s_{2}}{k_{i_{2}}}\right)^{2}\right)^{2}} \\
& v_{4}=\frac{k_{s_{1}} \mu_{1 \max } s_{1}}{\left(s_{1}+k_{s_{1}}\right)^{2}} \quad v_{11}=\frac{\left(k_{s_{2}}+\left(\frac{s_{2}}{k_{i_{2}}}\right)^{2}\right) \mu_{2 \max s_{2}}}{\left(s_{2}+k_{s_{2}}+\left(\frac{s_{2}}{k_{i_{2}}}\right)^{2}\right)^{2}} \\
& v_{5}=\frac{D(t) s_{2 i n}}{s_{2}} \quad v_{12}=\frac{2 \mu_{2 \max } s_{2}^{3}}{k_{i_{2}}^{2}\left(s_{2}+k_{s_{2}}+\left(\frac{s_{2}}{k_{i_{2}}}\right)^{2}\right)^{2}} \\
& v_{6}=\frac{k_{2} \mu_{1 \max } s_{1}^{2} x_{1}}{s_{2}\left(s_{1}+k_{s_{1}}\right)^{2}} \quad g=\frac{s_{2} e^{-e_{3}}+k_{s_{2}}+\left(\frac{s_{2}}{k_{i_{2}}}\right)^{2} e^{-2 e_{3}}}{s_{2}+k_{s_{2}}+\left(\frac{s_{2}}{k_{i_{2}}}\right)^{2}} \\
& v_{7}=\frac{k_{2} \mu_{1 \max } s_{1} x_{1}}{s_{2}\left(s_{1}+k_{s_{1}}\right)} \quad f_{3}=\frac{s_{2}+k_{s_{2}}+\left(\frac{s_{2}}{k_{i_{2}}}\right)^{2}}{s_{2} e^{-e_{3}}+k_{s_{2}}+\left(\frac{s_{2}}{k_{i_{2}}}\right)^{2} e^{-2 e_{3}}} \\
& f_{1}=\frac{s_{1}+k_{s_{1}}}{s_{1} e^{-e_{1}}+k_{s_{1}}} \quad f_{4}=\frac{s_{2}}{s_{2} e^{-e_{3}+k_{s_{2}}+\left(\frac{s_{2}}{k_{i_{2}}}\right)^{2} e^{-2 e_{3}}}} \\
& f_{2}=\frac{s_{1}}{s_{1} e^{-e_{1}}+k_{s_{1}}} \quad f_{5}=\frac{s_{2}^{2}}{k_{i_{2}}^{2}\left(s_{2} e^{-e_{3}}+k_{s_{2}}+\left(\frac{s_{2}}{k_{i_{2}}}\right)^{2} e^{-2 e_{3}}\right)}
\end{aligned}
$$

\section{REFERENCES}

[1] N. Aghannan and P. Rouchon. On Invariant Asymptotic Observers. In In Proceedings of the 41st IEEE Conference on Decision and Control, 2002.

[2] G. Bastin and D. Dochain. On-line Estimation and Adaptive Control of Bioreactors. Process measurement and control. Elsevier, 1990.

[3] O. Bernard and J.L Gouzé. Closed loop observers bundle for uncertain biotechnological models. Technical report.

[4] I. Didi. Sur l'Observation des Systmes Non Linaires Invariants: Application aux Bioprocédés. PhD thesis, Université AbouBekr Belkaid.

[5] Ibtissem Didi, Hacen Dib, and Brahim Cherki. An invariant observer for a chemostat model. Automatica, 50(9):2321-2326, 2014.

[6] D. Dochain A. Genovesi O. Bernard, Z. Hadj-Sadok and J.P. Steyer. Dynamical Model Development and Parameter Identification for an Anaerobic Wastewater Treatment Process. Biotechnology and Bioengineering, 75(4):424 - 438, 2001.

[7] P.J. Olver. Equivalence, Invariants and Symmetry. London Mathematical Society Lecture Note. Cambridge University Press, 1995.

[8] P. Martin S. Bonnabel and P. Rouchon. Symmetry-preserving observers. ArXiv Mathematics e-prints. 